\title{
Effects of Temperature and Water-Filtered Infrared-A Alone or in Combination on Healthy and Glyoxal-Stressed Fibroblast Cultures
}

\author{
Lilla Knels, ${ }^{1}$ Monika Valtink, ${ }^{1}$ Jamlec De la Vega Marin, ${ }^{1}$ Gerald Steiner, ${ }^{2}$ Cora Roehlecke, ${ }^{1}$ \\ Alexander Krueger, ${ }^{2}$ and Richard H. W. Funk ${ }^{1,3}$ \\ ${ }^{1}$ Institute of Anatomy of the Faculty of Medicine, TU Dresden, 01307 Dresden, Germany \\ ${ }^{2}$ Medical Sensoring and Monitoring of the Faculty of Medicine Carl Gustav Carus, TU Dresden, 01307 Dresden, Germany \\ ${ }^{3}$ CRTD/DFG-Center for Regenerative Therapies Dresden-Cluster of Excellence, TU Dresden, 01307 Dresden, Germany \\ Correspondence should be addressed to Lilla Knels, lilla.knels@tu-dresden.de
}

Received 10 August 2012; Revised 25 September 2012; Accepted 25 September 2012

Academic Editor: Elisa Cabiscol

Copyright ( $) 2012$ Lilla Knels et al. This is an open access article distributed under the Creative Commons Attribution License, which permits unrestricted use, distribution, and reproduction in any medium, provided the original work is properly cited.

\begin{abstract}
Water-filtered infrared-A (wIRA) radiation has been described as supportive for tissue regeneration. We sought to investigate in detail the wIRA effect at different temperatures in 3T3 fibroblasts that were treated with glyoxal to induce formation of advanced glycation end products (AGEs). Nonirradiated and nonglyoxal-treated cells served as controls. Experiments were carried out over a range of $37^{\circ}-45^{\circ} \mathrm{C}$ with exact temperature monitoring to distinguish between temperature and wIRA effects. Metabolic activity was assessed by resazurin assay. Mitochondrial membrane potential was assessed by JC-1 vital staining. Apoptotic changes were determined by vital staining with annexin V and YO-PRO-1 and determination of subG1 DNA content. Temperature had a dominant effect overriding effects exerted by wIRA or glyoxal treatment. The number of apoptotic cells was significantly higher at $45^{\circ} \mathrm{C}$, while the percentage of healthy cells was significantly lower at $45^{\circ} \mathrm{C}$. WIRA irradiation itself or in combination with glyoxal treatment exerted no damaging effects on the fibroblasts at physiological $\left(37^{\circ}-40^{\circ} \mathrm{C}\right)$ or higher $\left(42^{\circ}-45^{\circ} \mathrm{C}\right)$ temperatures compared to untreated controls. Temperatures of $45^{\circ} \mathrm{C}$, which can occur during inappropriate application of infrared irradiation, damage cells even in the absence of wIRA or glyoxal application.
\end{abstract}

\section{Introduction}

Water-filtered infrared-A (wIRA) radiation was successfully applied in the clinic, particularly in supporting wound healing and tissue repair, as recently reviewed [1]. WIRA corresponds to a large part of the sun's heat radiation, which reaches the surface of the Earth after being filtered by water vapor in the atmosphere. Water absorbs IR radiation between 0.75 and $2.4 \mu \mathrm{m}$ at four discrete wavelengths ranges, two being within infrared-A, one being at the transition from infrared-A to $-\mathrm{B}$, and one being within infraredB (Figure 1(a), blue boxes). These absorption bands arise from overtones of $\mathrm{O}-\mathrm{H}$ stretching vibrations of free water molecules. Unfiltered infrared-A can cause an undesired thermal load to exposed tissue. WIRA can artificially be produced by radiators with the radiation of a halogen lamp passing through a cuvette filled with water. Typical wIRA radiators do not emit UV radiation and almost no infrared-B and $-\mathrm{C}$ radiations. Figure 1(a) shows the relative intensity of an infrared radiator equipped with a water cuvette as filter. Spectra were ratioed against the spectrum of the radiator without water cuvette. The intense water absorption bands around $0.96 \mu \mathrm{m}, 1.19 \mu \mathrm{m}$, between $1.4 \mu \mathrm{m}$ and $1.55 \mu \mathrm{m}$ as well as over $1.8 \mu \mathrm{m}$ are clearly visible. Due to the strong absorption of water, no light between 1.4 and $2.4 \mu \mathrm{m}$ passed the water cuvette.

Although numerous publications demonstrated the beneficial effects of wIRA irradiation in vivo on injured tissues and in vitro on cell cultures [2-5], other studies suggested that wIRA irradiation is detrimental to cell cultures [6]. However, these studies did not adequately address the influence of mere temperature increases which are possibly 


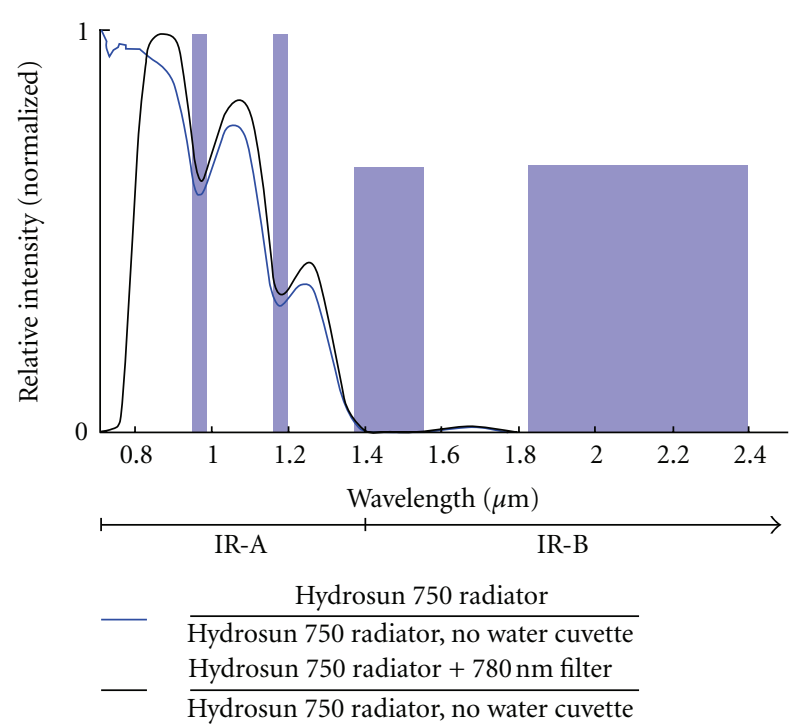

(a)

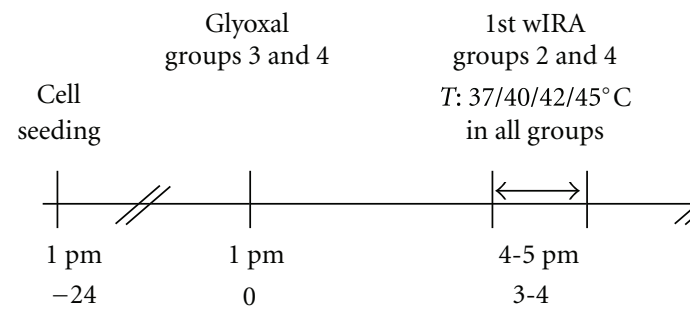

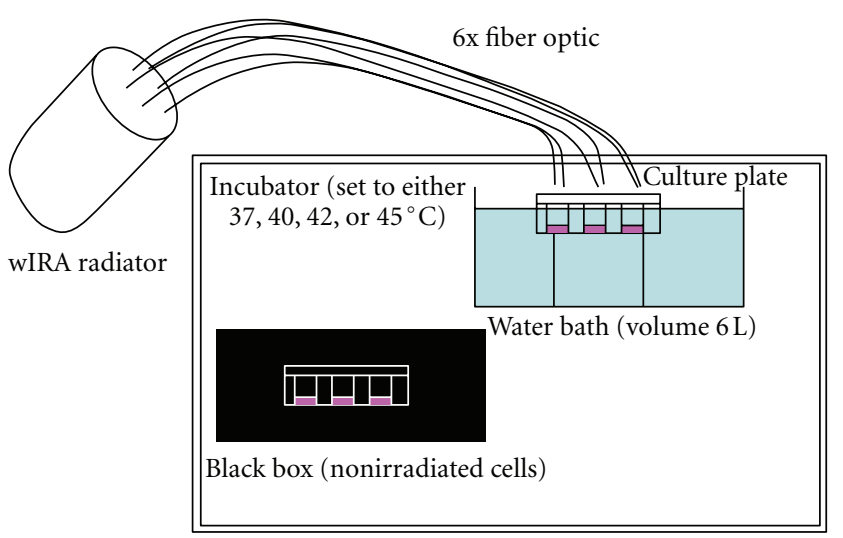

(b)

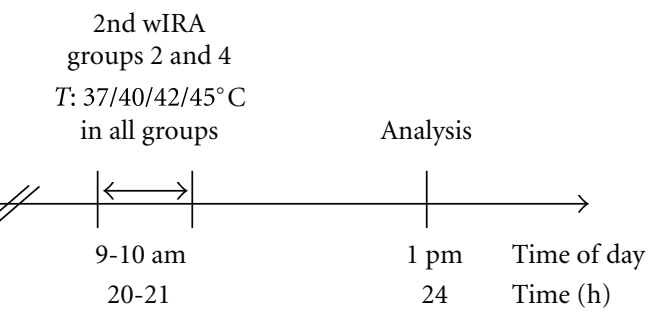

(c)

FIgURE 1: Experimental conditions. (a) Transmission spectra of the Hydrosun 750 radiator with and without $750 \mathrm{~nm}$ cutoff filter. The reference spectrum was recorded from the same radiator without a water cuvette. Spectra are normalized to their maximal transmission. (b) A cell incubator was equipped with a wIRA radiator, whose radiation was guided by optical fibres into the incubator directly onto the cell culture plates ( $4 \mathrm{~cm}$ above the plates). The plates were placed in a large water bath (6 liters) to facilitate maintenance of constant temperatures during irradiation. (c) Cultures were allocated to four different treatment groups: group 1 control without wIRA, group 2 control with wIRA, group 3 glyoxal without wIRA, group 4 glyoxal with wIRA. Cells were either irradiated with wIRA or kept nonirradiated at the indicated temperatures and times after addition of glyoxal.

evoked by wIRA and may cause damages in healthy or sublethally stressed cells. Such temperature-evoked damages may add to or even multiply with possible detrimental effects caused by wIRA, for example, when applied inappropriately. In combination with metabolic stress, these potential adverse effects may aggravate any deleterious effects of wIRA and/or temperature rises in cells or tissues. In vitro, metabolic stress can be induced by glyoxal. Glyoxal is an intermediate product in the glycation reaction and causes the formation of altered proteins and DNA damage products, thereby mimicking the situation of stressed cells in diabetic metabolic status. We have previously shown that glyoxal leads to an accelerated production of advanced glycation end products (AGEs), inducing cellular damage and apoptosis [7]. Glyoxal treatment has also been shown to induce senescence in cultured human fibroblasts [8].

In the present study, the effects of wIRA irradiation on healthy fibroblasts or fibroblasts damaged by glyoxal were assessed at different temperatures. Glyoxal exposure was used in order to induce a diabetic metabolic status in the cells.
Using a sublethal concentration of glyoxal, which stresses the cells but does not induce cell death, we were able to discriminate between damages evoked by glyoxal, wIRA, temperature, or a combination of these. This study shows that wIRA itself exerts no damaging effect to cells, while the damaging effect of high temperature even overrides that of glyoxal.

\section{Materials and Methods}

2.1. Irradiation Setup. A cell incubator was equipped with an external wIRA radiator (Hydrosun 750, Hydrosun, Müllheim, Germany; approximately 600-1400 nm) with optical fibers leading the radiation into the incubator (Figure 1(b)). The radiator was equipped with a filter blocking wavelengths below $780 \mathrm{~nm}$ to exclude effects due to visible light. The emitters were placed $4 \mathrm{~cm}$ above the cell culture plates. The plates were continuously placed in a thermostatically controlled water bath (6 liter), because water has a better conduction of heat than air. Temperature 
changes at the bottom of the culture plates were monitored with a temperature sensor which was covered with a $9 \mathrm{~mm}^{2}$ piece of aluminum foil without touching it to protect the sensor from wIRA. Irradiation intensity was measured with a radiophotometer (model HBM1, Hydrosun) and set to deliver $30 \mathrm{~mW} / \mathrm{cm}^{2}$ at the bottom of the plates, corresponding to clinically applied wIRA radiation of 60$120 \mathrm{~mW} / \mathrm{cm}^{2}$ [9]. WIRA irradiation was applied under temperature-controlled conditions at $37^{\circ} \mathrm{C}, 40^{\circ} \mathrm{C}, 42^{\circ} \mathrm{C}$, and $45^{\circ} \mathrm{C}$. Nonirradiated control cells were placed in the same incubator inside a black box to exclude any irradiation under otherwise same conditions (Figure 1(b)). Before and after wIRA irradiation, all cells were incubated in a second cell culture incubator at $37^{\circ} \mathrm{C}\left(5 \% \mathrm{CO}_{2}, 96 \%\right.$ humidity $)$.

2.2. Spectroscopic Measurements. Spectra of the "Hydrosun 750 radiator" were recorded with a conventional Fouriertransform NIR spectrometer (Vector 22/N, Bruker Optik $\mathrm{GmbH}$, Ettlingen, Germany). The radiation was coupled into the spectrometer by using the port for external light sources. All spectra were measured with $4 \mathrm{~cm}^{-1}$ resolution and 100 scans by the deuterated triglycine sulfate (DTGS) detector. The spectrum of the Hydrosun 750 radiator without water cuvette was used as reference. Ratioed spectra were normalized to maximum intensity.

2.3. Temperature Adjustment and Monitoring. $24 \mathrm{~h}$ before experiments, the radiator was switched on. A culture plate with medium was placed in the water bath and irradiated with wIRA. A sensor was placed at the bottom of a well, and temperature was monitored continuously with a precision of $\pm 0.02^{\circ} \mathrm{C}$ using an electronic measuring device (ALMEMO Model MA2590-4S, Ahlborn, Germany). The incubator temperature was set so as to factor in WIRAinduced temperature rises, and short-term variabilities were balanced via temperature regulation of the water bath. During experiments, one well of the culture plate was filled with medium but left without cells, and temperatures were monitored accordingly with a small sensor, allowing to differentiate between the effects of heat and wIRA radiation.

2.4. Cell Culture. 3T3 cells were cultured in MEM (Minimum Essential Medium Eagle, w/L-glutamine, PAN Biotech, Aidenbach, Germany) supplemented with 5\% FCS (fetal calf serum, Biochrom, Berlin, Germany). Cells were seeded at 25,000 cells $/ \mathrm{cm}^{2}$ onto 6 -well plates $24 \mathrm{~h}$ before incubation in glyoxal (see Figure 1(c) for experimental outline). Control cells were incubated either without (normal metabolic state) or with $1 \mathrm{mmol} / \mathrm{L}$ glyoxal (Sigma, Deisenhofen, Germany) to induce sublethal metabolic stress (diabetic metabolic state). At 3 and 20 hours after glyoxal addition, cells were either irradiated for $1 \mathrm{~h}$ with $30 \mathrm{~mW} / \mathrm{cm}^{2}$ wIRA (total dose $216 \mathrm{~J} / \mathrm{cm}^{2}$ in $24 \mathrm{~h}$ ), or remained nonirradiated, resulting in four different treatment groups: (1) control without wIRA, (2) control with wIRA, (3) glyoxal without wIRA, and (4) glyoxal with wIRA. During wIRA irradiation, the temperature of the incubator was set to either $37^{\circ} \mathrm{C}, 40^{\circ} \mathrm{C}$, $42^{\circ} \mathrm{C}$, or $45^{\circ} \mathrm{C}$ to mimic thermal stress, which might occur during inappropriate clinical application of wIRA. The nonirradiated control cells were likewise exposed to thermal stress.

2.5. Cytotoxicity/Metabolic Activity Assay. Resazurin, which is converted to resorufin by intracellular redox systems, was used to determine metabolic activity of cells. Cells were seeded at 25,000 cells $/ \mathrm{cm}^{2}$ onto 96-well plates and treated with glyoxal and/or wIRA under same conditions as described previously (Figure 1(c)). Resazurin (Sigma, Deisenhofen, Germany) was added $20 \mathrm{~h}$ after addition of glyoxal according to the manufacturer's instructions. Wells without cells but containing medium and resazurin were used as blanks. Absorbance was measured at $600 \mathrm{~nm}$ after $4 \mathrm{~h}$ using a plate reader (Model Sunrise, Tecan, Austria). Results are given as difference in absorbance (absorbance blank $_{-}$ absorbance sample $)$.

2.6. Determination of Mitochondrial Membrane Potential. Cells were seeded at 25,000 cells $/ \mathrm{cm}^{2}$ into 8 -chamber slides (Labtek coverglass system, Nunc, New York, USA) and treated with glyoxal and/or wIRA under same conditions as described above (Figure 1(c)). Vital staining was performed with JC-1 $(20 \mu \mathrm{g} / \mathrm{mL}$ medium; Invitrogen $)$ at $37^{\circ} \mathrm{C}$ for 20 $30 \mathrm{~min}$. Samples were immediately photodocumented using an IX81 fluorescence microscope equipped with an OBS FV2T camera driven by cell ${ }^{\wedge} \mathrm{R}$ software (all by Olympus, Hamburg, Germany). Red J-aggregates were recorded using a $536 \pm 20 \mathrm{~nm} / 610 \pm 36 \mathrm{~nm}$ filter pair, and green JC-1 monomers were recorded with a $486 \pm 17 \mathrm{~nm} / 540 \pm 25 \mathrm{~nm}$ filter pair.

2.7. Determination of Necrosis and Apoptosis. Necrotic and apoptotic cells were detected simultaneously by vital staining with propidium iodide for necrotic cells, and either annexin V or YO-PRO-1 for apoptotic cells. 10,000 cells were analyzed by flow cytometry in a FACS Calibur (Becton Dickinson, Franklin Lakes, USA). The percentages of necrotic and apoptotic cells were calculated with BD CellQuest software.

FITC-conjugated annexin V (ImmunoTools, Friesoythe, Germany) was diluted with binding buffer containing $10 \mathrm{mmol} / \mathrm{L}$ HEPES (N-2-hydroxyethylpiperazine- $\mathrm{N}^{\prime}$-2ethanesulfonic acid), $\mathrm{pH} 7.4,140 \mathrm{mmol} / \mathrm{L} \mathrm{NaCl}$, and $2.5 \mathrm{mmol} / \mathrm{L} \mathrm{CaCl}_{2}$. Cells were harvested and incubated with $50 \mu \mathrm{L}$ annexin $\mathrm{V}$ solution and $1 \mu \mathrm{L}$ propidium iodide solution $(1 \mathrm{mg} / \mathrm{mL}$ in distilled water) for $5 \mathrm{~min}$ at room temperature in the dark. Cells were analyzed by flow cytometry using a $530 \pm 15 \mathrm{~nm}$ filter for annexin $\mathrm{V}$ and a $585 \pm 21 \mathrm{~nm}$ filter for propidium iodide.

For YO-PRO-1 staining, cells were harvested and incubated in $50 \mu \mathrm{L}$ PBS with $1 \%$ horse serum (Brand, Wertheim, Germany) containing $1 \mu \mathrm{L}$ YO-PRO- $1 \quad(100 \mu \mathrm{mol} / \mathrm{L}$ in DMSO) and $1 \mu \mathrm{L}$ propidium iodide $(1 \mathrm{mg} / \mathrm{mL}$ in distilled water) for $20 \mathrm{~min}$ at room temperature in the dark. Flow cytometric analysis was performed using a $530 \pm 15 \mathrm{~nm}$ filter for YO-PRO- 1 and a $585 \pm 21 \mathrm{~nm}$ filter for propidium iodide.

Apoptotic cells were also detected by nuclear staining of fixed cells to quantify the number of cells with a DNA 
content less than in G1 phase of the cell cycle (subG1-DNA content). Cells were harvested and fixed in $70 \%$ ethanol for $5 \mathrm{~min}$ at room temperature. $50 \mu \mathrm{L}$ phosphate-buffered saline (PBS) containing $1 \mu \mathrm{L}$ propidium iodide solution $(1 \mathrm{mg} / \mathrm{mL}$ in distilled water) and $1 \mu \mathrm{L}$ RNase $(>7 \mathrm{U} / \mu \mathrm{L}$, Roth, Karlsruhe, Germany) were added to the cells, and samples were incubated for $30 \mathrm{~min}$ at room temperature in the dark. Cells with apoptotic nuclei were detected by flow cytometry using a $585 \pm 21 \mathrm{~nm}$ filter, yielding a broad subdiploid peak that could easily be distinguished from the narrow peak of nonapoptotic, diploid cells. The percentage of apoptotic cells was calculated from the histograms.

2.8. Statistical Analysis. All assays were performed at least six times. Values are given as mean \pm SEM. Statistical analysis was performed by multifactorial univariate analysis of variance (multiway ANOVA) followed by post hoc analysis (Bonferroni-test). Alpha error was adjusted appropriately, and significance was accepted at $P<0.05$. Statistical analysis was performed using SPSS 17.0 (Chicago, USA).

\section{Results}

3.1. Metabolic Activity and Mitochondrial Membrane Potential. Metabolic activity was determined using the resazurin conversion assay. Resazurin is a substrate that undergoes a colour change in response to reduction as an indicator of cell metabolic activity. Using this assay, it was observed that the metabolic activity of cells was drastically lower at $45^{\circ} \mathrm{C}$ with high statistical significance in comparison to the metabolic activity of cells cultured at the other temperatures (Figure 2, bar chart). Metabolic activity of cells was also significantly higher at $42^{\circ} \mathrm{C}$ than at $37^{\circ} \mathrm{C}$.

The vital dye JC-1 permeates cell membranes and selectively accumulates within the mitochondrial matrix. It forms red fluorescent J-aggregates in the presence of a highly negative mitochondrial membrane potential but exists as green monomers under depolarized conditions. Staining live cells with JC-1 revealed that wIRA irradiation, incubation with glyoxal, or both in combination did not have a distinct effect on the cells at $37^{\circ} \mathrm{C}$ (Figure 2, micrograph panel). When temperature was risen to $45^{\circ} \mathrm{C}$, all mitochondria appeared depolarized irrespective of wIRA irradiation or glyoxal treatment, indicating that temperature exerts a dominant effect on mitochondrial membrane potential (Figure 2, micrograph panel).

3.2. Necrosis and Apoptosis. Propidium iodide is membrane impermeant and can only enter cells that have damaged membranes. It accumulates in the nucleus and intercalates with nucleic acid, thereby emitting a characteristic red fluorescence. Hence, vital staining with propidium iodide allows to determine the number of necrotic cells. The number of propidium iodide-positive necrotic cells was significantly higher in cultures that were kept at $45^{\circ} \mathrm{C}$ than in cultures grown at the other temperatures (Figure 3(a)), confirming results obtained by resazurin conversion and JC1 staining. Effects of glyoxal and/or wIRA were neglectable compared to that of temperature and did not influence the cells significantly, indicating that temperature exerts a dominant effect.

Phosphatidylserine, which is found exclusively on the inner leaflet of the cell membrane in healthy cells, is translocated to the outer cell membrane leaflet during early stages of apoptosis. Annexin V binds specifically to phosphatidylserine and can be used to determine apoptotic cells by microscopy and flow cytometry. Since Annexin V can also enter necrotic cells and then bind to phosphatidylserine at the inner leaflet, necrotic cells must be discriminated from apoptotic cells by simultaneous labelling with propidium iodide. Figure 3(b) shows the intensity of annexin V fluorescence in propidium iodide-negative cells, which was significantly higher at $45^{\circ} \mathrm{C}$ than at the other temperatures. This sensitive method further revealed that wIRA did not have a significant effect on the cells.

Nuclear membranes of healthy cells are impermeable to YO-PRO-1, but this dye easily penetrates the nuclear membranes of apoptotic and necrotic cells. It then intercalates with DNA and emits a characteristic green fluorescence, thereby allowing accurate and reproducible quantification of dying cells. Double labelling with YO-PRO-1 and propidium iodide under vital conditions reveals apoptotic cells by green fluorescence and necrotic cells by red and green fluorescence, while viable cells remain nonfluorescent. The percentage of YO-PRO-1-positive, propidium iodide-negative cells was significantly different between cells treated at $37^{\circ} \mathrm{C}$ and cells treated at $45^{\circ} \mathrm{C}$ but was not significant compared to the other temperatures. WIRA irradiation significantly decreased the percentage of apoptotic, YO-PRO-1 positive cells irrespective of the temperature, while glyoxal exerted no significant effect (Figure 3(c)).

Labelling of fixed cells with propidium iodide allows to determine the DNA content in each cell in correlation with fluorescence intensity and can be used to perform cell cycle analysis. Apoptotic cells with fragmented DNA can be identified by their subG1-DNA content. The percentage of cells with sub-G1-DNA content was significantly higher at $45^{\circ} \mathrm{C}$ than at the other temperatures and was significantly lower at $42^{\circ} \mathrm{C}$ than at $37^{\circ} \mathrm{C}$ (Figure $3(\mathrm{~d})$ ). The effect exerted by temperature was dominant so that, irrespective of temperature, any significant effects of glyoxal and/or wIRA were not observed.

\section{Discussion}

Infrared-A radiation was shown to accelerate cell and tissue repair in vivo [10], to improve recovery from infected, ischemic, hypoxic, and toxic injury [11-15], and to reduce degeneration of the injured optic nerve or traumatic brain injury $[16,17]$. Previous studies and reviews reported on successful clinical applications of wIRA radiation in patients with acute and chronic wounds, sclerodermia, local fat reduction, or actinic keratosis $[1,4,18-20]$. It was also demonstrated that irradiation with red light $(630 \mathrm{~nm})$ may assist in healing of diabetic wounds [11]; hence, it may also 


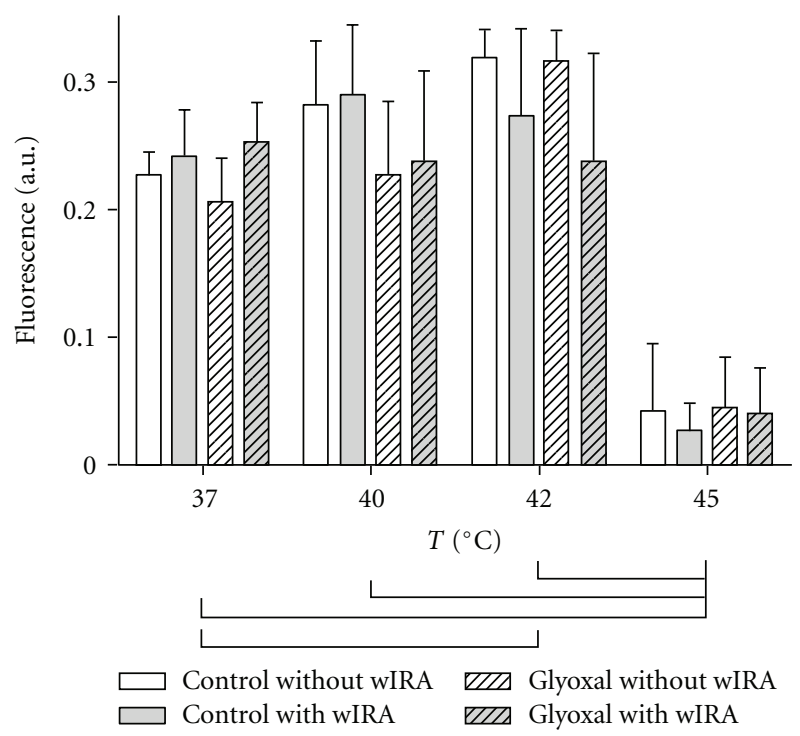

(a)
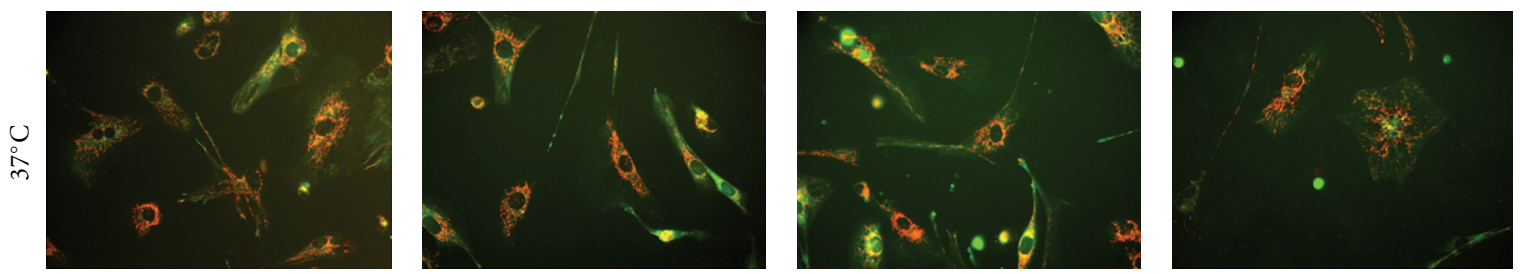

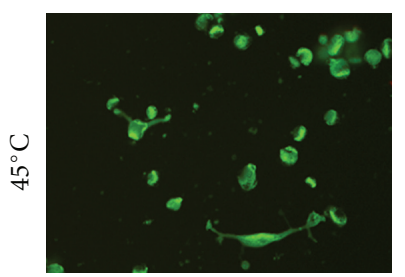

Control without wIRA

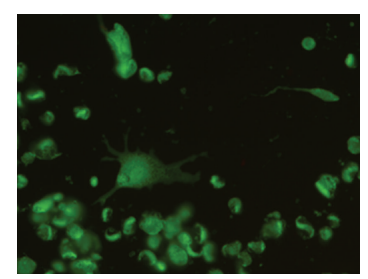

Control with wIRA

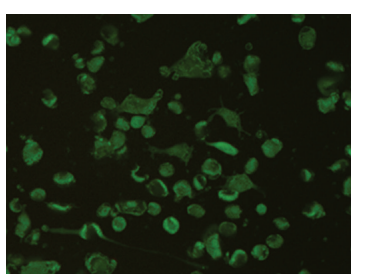

Glyoxal without wIRA

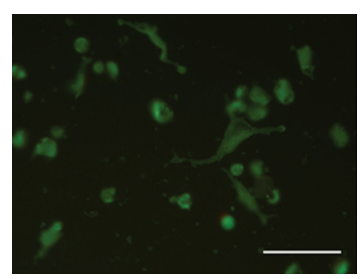

Glyoxal with wIRA

(b)

FIgURE 2: Metabolic activity. The bar chart shows metabolic activity of the cells as determined by resazurin conversion assay at different temperatures and after treatment with or without glyoxal and/or wIRA. Squared brackets indicate statistically significant differences $(P<$ 0.05 ) between temperatures. Cells were able to maintain adequate metabolic activity at temperatures up to $42^{\circ} \mathrm{C}$, but their metabolic activity declined significantly at $45^{\circ} \mathrm{C}$. The micrographs show cells that were stained with JC-1 for visualising the mitochondrial membrane potential. Red fluorescence, as seen in cells cultured at $37^{\circ} \mathrm{C}$, indicates a highly negative mitochondrial membrane potential, while green fluorescence, as seen in cells cultured at $45^{\circ} \mathrm{C}$, indicates that the mitochondrial membranes were depolarised. No differences between the treatment groups (with or without glyoxal and/or wIRA) could be observed. Scale bar: $100 \mu \mathrm{m}$.

be assumed that treatment with wIRA can exert beneficial effects on such impaired wound healing.

The present study was performed to investigate the effects of wIRA on fibroblasts in more detail under normal and metabolically stressed conditions because fibroblasts play a major role in connective tissue and wound repair. Recently, contradictory studies were published which demonstrated either positive effects of wIRA on fibroblasts [21, 22], or showed adverse cellular responses of cultured foreskin fibroblasts to wIRA irradiation which the authors attributed to the infrared radiation [6]. However, neither of the authors differentiated between thermoneutral effects of wIRA and effects induced by temperature changes. An important aim of our study was therefore to differentiate between the effects of wIRA alone and those due to temperature increases that might occur during wIRA irradiation either because of inappropriate application of wIRA or independently of wIRA $[21,23]$. In our experiments, temperature was constantly monitored and carefully controlled by using an electronic measuring device with sensors situated at the bottom of the culture plates in the medium in a cell-free well. In addition, the cell culture plates were placed in a large water bath, because water reacts slower to temperature shifts than air and facilitates to hold a constant temperature during irradiation. We used 3T3 mouse fibroblasts, a well-defined and wellcharacterized fibroblast cell line with a stable growth rate. 


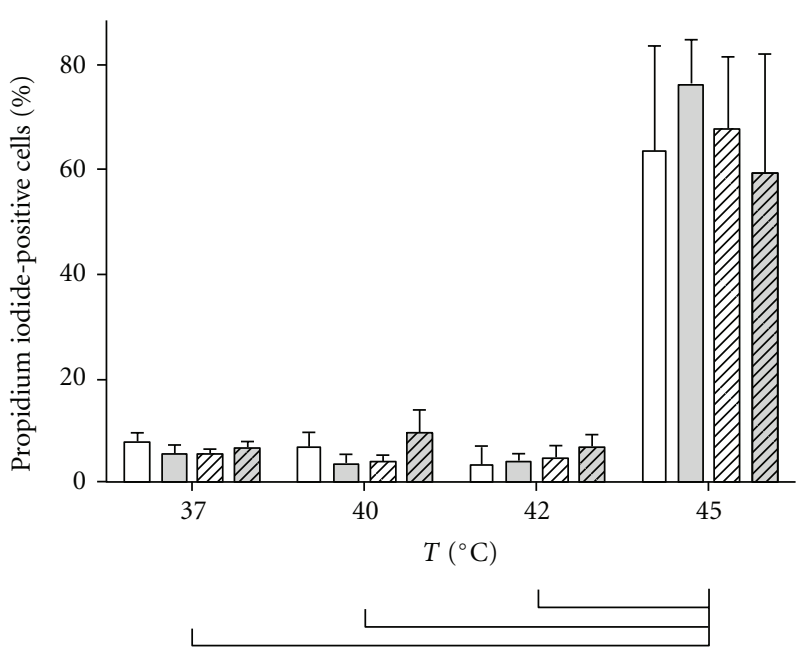

(a)

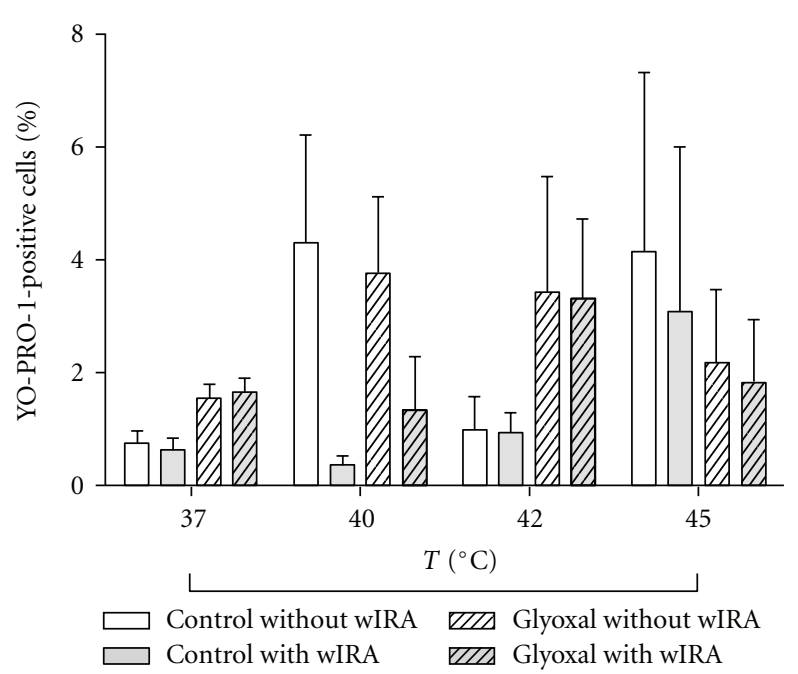

(c)

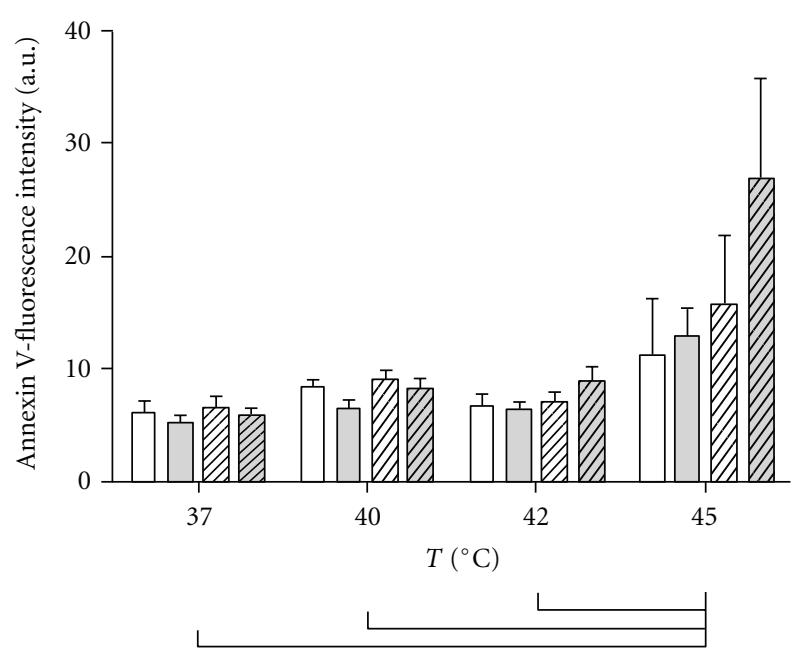

(b)

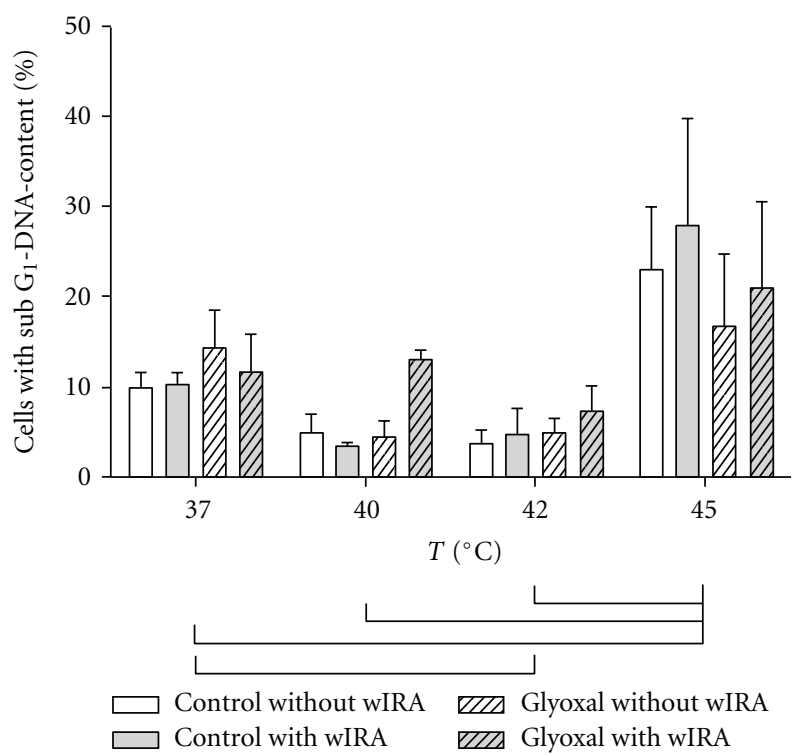

(d)

Figure 3: Necrosis and apoptosis. Cells were treated with or without glyoxal and/or wIRA at indicated temperatures and were subdued to vital staining with propidium iodide (a), annexin $\mathrm{V}(\mathrm{b})$, and YO-PRO-1 (c), as well as staining of fixed cells with propidium iodide for cell cycle analysis $(\mathrm{d})$. Squared brackets indicate statistically significant differences $(P<0.05)$ between temperatures. (a) The percentage of necrotic cells (propidium iodide positive) was significantly higher at $45^{\circ} \mathrm{C}$ than at the other temperatures but was not affected by glyoxal and/or wIRA treatment. (b) The percentage of apoptotic (annexin V positive) cells was also significantly higher at $45^{\circ} \mathrm{C}$ than at the other temperatures. (c) The number of apoptotic cells determined by vital staining with YO-PRO-1 was significantly higher at $45^{\circ} \mathrm{C}$ than at $37^{\circ} \mathrm{C}$. (d) Cell cycle analysis revealed that the number of cells with subG1 DNA-content was significantly higher at $45^{\circ} \mathrm{C}$ than at the other temperatures and was also significantly higher at $37^{\circ} \mathrm{C}$ than at $42^{\circ} \mathrm{C}$.

Some of the fibroblast cultures were stressed by exposure to glyoxal in order to investigate the effects of wIRA radiation on fibroblast cells undergoing oxidative stress similar to that in a diabetic metabolic state.

It could be observed that adverse effects occurred independently of wIRA irradiation and were also seen in nonirradiated cells. The experiments revealed that temperature exerted a dominant effect on the cells and turned out to be the only significantly influencing factor. At physiological temperatures of $37^{\circ} \mathrm{C}$ and at $40^{\circ} \mathrm{C}$, no damaging effects on cultured fibroblasts were observed irrespective of whether wIRA was applied or not. At higher temperatures of $42^{\circ} \mathrm{C}$ and $45^{\circ} \mathrm{C}$, negative cellular responses were observed which occurred in every treatment group, that is, independent of whether wIRA and/or glyoxal were applied or not, and were attributable solely to thermal effects. This is in agreement with the results of a study which showed a beneficial effect of IRA on primary neuronal cell cultures [12], and another 
study which showed no damaging effects of wIRA on human fibroblasts even at high irradiances as long as appropriate temperatures were not exceeded [23]. Furthermore, it was shown that the surface of human skin exhibits surface temperatures of only $37^{\circ}-39^{\circ} \mathrm{C}$ when wIRA is applied appropriately even at high irradiances $[5,24]$. In other experiments carried out at high temperatures, deleterious effects were shown to be independent and irrespective of the application of wIRA [23]. The temperature in subcutaneous and other connective tissues does not exceed skin surface temperature under irradiation with wIRA, so that wIRA, when applied at appropriate therapeutic irradiances and doses, does not result in critical temperature increases [2].

We could previously show that glyoxal induces the formation of AGEs, leading to cellular damage and apoptosis in cell cultures [7]. Here, the production of AGEs was induced by glyoxal exposure in order to mimic the conditions of fibroblasts under sublethal oxidative stress similar to that seen in diabetic patients. The effects of the cell stressor glyoxal either alone or in combination with wIRA radiation on cultured fibroblasts at $37^{\circ}-45^{\circ} \mathrm{C}$ were investigated with respect to key features of apoptosis. The dominating effect of a high temperature of $45^{\circ} \mathrm{C}$ was significant compared to other effects that may have been provoked by glyoxal and/or wIRA, or were seen at lower temperatures. In fact, the damage induced by high temperature was seen in glyoxaland/or wIRA-treated cells as well as in untreated cells at comparable extent. Furthermore, it seemed that the negative effect of high temperature was overriding and thereby masking any effects of glyoxal and of wIRA, as indicated by the lack of significant differences between the four treatment groups at $45^{\circ} \mathrm{C}$.

The slight increase in metabolic activity over the range of $37^{\circ} \mathrm{C}$ to $42^{\circ} \mathrm{C}$ is in accordance with the general reaction velocity rule valid in chemical and biochemical reactions, whereas at $45^{\circ} \mathrm{C}$, the damaging thermal effect dominated. This is also in agreement with the thermal working principles of wIRA. These include a higher metabolic rate and a higher energy production in vitro based on increased temperature as well as an unspecific absorption of electromagnetic radiation by intracellular water, which is significantly lower for wIRA than for IRA (Figure 1(a)) $[23,25]$. In vivo, the higher metabolic rate is furthermore based on increased partial pressure of oxygen and higher perfusion of the tissue when exposed to wIRA [2]. WIRA irradiation itself did not exert deleterious effects and did not aggravate any glyoxal-induced damage in in vitro cultured fibroblasts. On the contrary, a positive effect was seen when cells were stained with YO-PRO-1, indicating that wIRA irradiation might reduce the number of apoptotic cells. However, since temperature effects were dominant and overrode any other possible effects in most of the experiments, such positive effects of wIRA remain to be proven in further studies.

\section{Disclosures}

The authors indicate no financial interest.

\section{Acknowledgments}

The authors wish to thank E. Kuhlisch, Institute of Medical Informatics and Biometry (IMB) of the Faculty of Medicine of the TU Dresden, for support in statistical analysis, and W. Müller, Dr. med. h.c. Erwin Braun Stiftung, and H. Piazena, Medical Photobiology Group, Charité Universitätsmedizin Berlin, for technical support. This study was supported by a grant from the Dr. med. h.c. Erwin Braun Stiftung, Basel, Switzerland.

\section{References}

[1] G. Hoffmann, "Water-filtered infrared-A (wIRA) in acute and chronic wounds," German Medical Science Krankenhaushygiene Interdisziplinär, vol. 4, Doc12, no. 2, 2009.

[2] M. Hartel, G. Hoffmann, M. N. Wente, M. E. Martignoni, M. W. Büchler, and H. Friess, "Randomized clinical trial of the influence of local water-filtered infrared A irradiation on wound healing after abdominal surgery," British Journal of Surgery, vol. 93, no. 8, pp. 952-960, 2006.

[3] M. Hartel, P. Illing, J. B. Mercer, J. Lademann, G. Daeschlein, and G. Hoffmann, "Therapy of acute wounds with waterfiltered infrared-A (wIRA)," German Medical Science Krankenhaushygiene Interdisziplinär, vol. 2, Doc52, no. 2, 2008.

[4] V. von Felbert, G. Hoffmann, S. Hoff-Lesch et al., "Photodynamic therapy of multiple actinic keratoses: reduced pain through use of visible light plus water-filtered infrared A compared with light from light-emitting diodes," British Journal of Dermatology, vol. 163, no. 3, pp. 607-615, 2010.

[5] J. B. Mercer, S. P. Nielsen, and G. Hoffmann, "Improvement of wound healing by water-filtered infrared-A (wIRA) in patients with chronic venous stasis ulcers of the lower legs including evaluation using infrared thermography," GMS German Medical Science, vol. 6, Doc11, 2008.

[6] P. Schroeder, C. Pohl, C. Calles, C. Marks, S. Wild, and J. Krutmann, "Cellular response to infrared radiation involves retrograde mitochondrial signaling," Free Radical Biology and Medicine, vol. 43, no. 1, pp. 128-135, 2007.

[7] L. Knels, M. Worm, M. Wendel, C. Roehlecke, E. Kniep, and R. H. W. Funk, "Effects of advanced glycation end productsinductor glyoxal and hydrogen peroxide as oxidative stress factors on rat retinal organ cultures and neuroprotection by UK-14,304," Journal of Neurochemistry, vol. 106, no. 4, pp. 1876-1887, 2008.

[8] H. Sejersen and S. I. S. Rattan, "Glyoxal-induced premature senescence in human fibroblasts," Annals of the New York Academy of Sciences, vol. 1100, pp. 518-523, 2007.

[9] H. Piazena and D. K. Kelleher, "Effects of infrared-A irradiation on skin: discrepancies in published data highlight the need for an exact consideration of physical and photobiological laws and appropriate experimental settings," Photochemistry and Photobiology, vol. 86, no. 3, pp. 687-705, 2010.

[10] C. Jantschitsch, S. Majewski, A. Maeda, T. Schwarz, and A. Schwarz, "Infrared radiation confers resistance to UV-induced apoptosis via reduction of DNA damage and upregulation of antiapoptotic proteins," Journal of Investigative Dermatology, vol. 129, no. 5, pp. 1271-1279, 2009.

[11] M. J. Conlan, "Biostimulation of wound healing by low-energy laser irradiation A review," Journal of Clinical Periodontology, vol. 23, no. 5, pp. 492-496, 1996. 
[12] M. T. T. Wong-Riley, H. L. Liang, J. T. Eells et al., "Photobiomodulation directly benefits primary neurons functionally inactivated by toxins: role of cytochrome c oxidase," Journal of Biological Chemistry, vol. 280, no. 6, pp. 4761-4771, 2005.

[13] P. Iordanou, E. G. Lykoudis, A. Athanasiou et al., "Effect of visible and infrared polarized light on the healing process of fullthickness skin wounds: an experimental study," Photomedicine and Laser Surgery, vol. 27, no. 2, pp. 261-267, 2009.

[14] W. Yu, J. O. Naim, and R. J. Lanzafame, "Effects of photostimulation on wound healing in diabetic mice," Lasers in Surgery and Medicine, vol. 20, no. 1, pp. 56-63, 1997.

[15] A. P. Sommer, A. L. B. Pinheiro, A. R. Mester, R. P. Franke, and H. T. Whelan, "Biostimulatory windows in low-intensity laser activation: lasers, scanners, and NASA's light-emitting diode array system," Journal of Clinical Laser Medicine and Surgery, vol. 19, no. 1, pp. 29-33, 2001.

[16] T. Ando, W. Xuan, T. Xu et al., "Comparison of therapeutic effects between pulsed and continuous wave $810 \mathrm{~nm}$ wavelength laser irradiation for traumatic brain injury in mice," PLoS ONE, vol. 6, Article ID e26212, 2011.

[17] K. D. Desmet, D. A. Paz, J. J. Corry et al., "Clinical and experimental applications of NIR-LED photobiomodulation," Photomedicine and Laser Surgery, vol. 24, no. 2, pp. 121-128, 2006.

[18] V. von Felbert, D. Simon, L. R. Braathen, M. Megahed, and T. Hunziker, "Treatment of linear scleroderma with waterfiltered infrared-A irradiation," Hautarzt, vol. 58, no. 11, pp. 923-924, 2007.

[19] F. Möckel, G. Hoffmann, R. Obermuller, W. Drobnik, and G. Schmitz, "Influence of water-filtered infrared-A (wIRA) on reduction of local fat and body weight by physical exercise," GMS German Medical Science, vol. 4, Doc05, 2006.

[20] V. von Felbert, H. Schumann, J. B. Mercer, W. Strasser, G. Daeschlein, and G. Hoffmann, "Therapy of chronic wounds with water-filtered infrared-A (wIRA)," German Medical Science Krankenhaushygiene Interdisziplinär, vol. 2, Doc 52, 2007.

[21] N. Gebbers, N. Hirt-Burri, C. Scaletta, G. Hoffmann, and L. A. Applegate, "Water-filtered infrared-A radiation (wIRA) is not implicated in cellular degeneration of human skin," GMS German Medical Science, vol. 5, Doc08, 2007.

[22] L. A. Applegate, C. Scaletta, R. Panizzon, E. Frenk, P. Hohlfeld, and S. Schwarzkopf, "Induction of the putative protective protein ferritin by infrared radiation: implications in skin repair.," International Journal of Molecular Medicine, vol. 5, no. 3, pp. 247-251, 2000.

[23] T. Jung, A. Höhn, H. Piazena, and T. Grune, "Effects of waterfiltered infrared A irradiation on human fibroblasts," Free Radical Biology and Medicine, vol. 48, no. 1, pp. 153-160, 2010.

[24] J. B. Mercer and L. De Weerd, "The effect of water-filtered infrared-A (wIRA) irradiation on skin temperature and skin blood flow as evaluated by infrared thermography and scanning laser Doppler imaging," Thermology International, vol. 15, no. 3, pp. 89-94, 2005.

[25] T. Jung and T. Grune, "Experimental basis for discriminating between thermal and athermal effects of water-filtered infrared A irradiation," Annals of the New York Academy of Sciences, vol. 1259, pp. 33-38, 2011. 


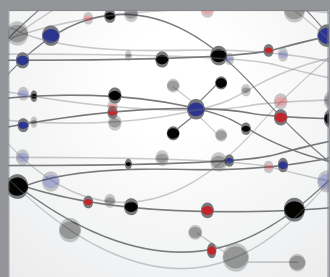

The Scientific World Journal
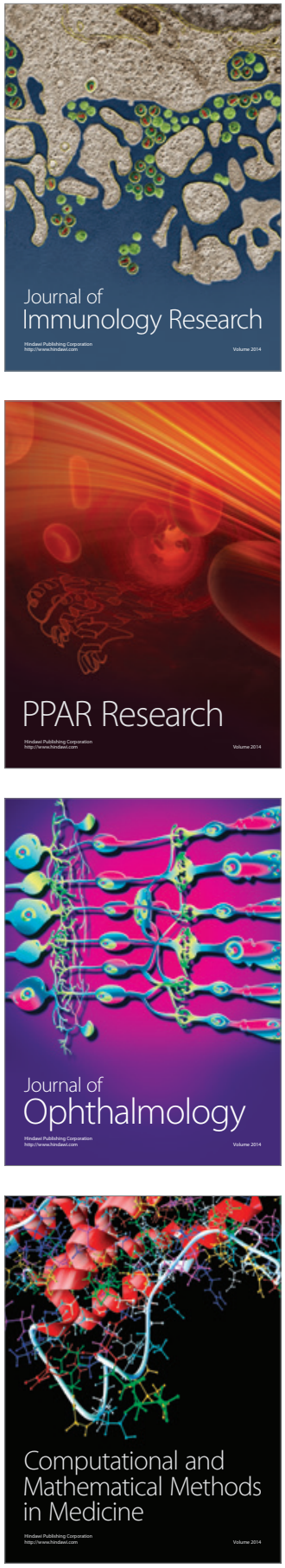

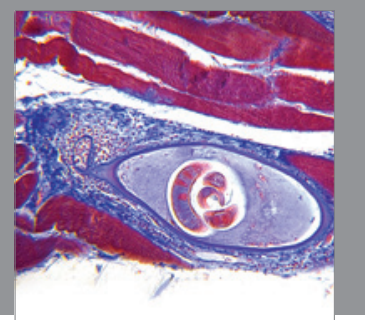

Gastroenterology

Research and Practice
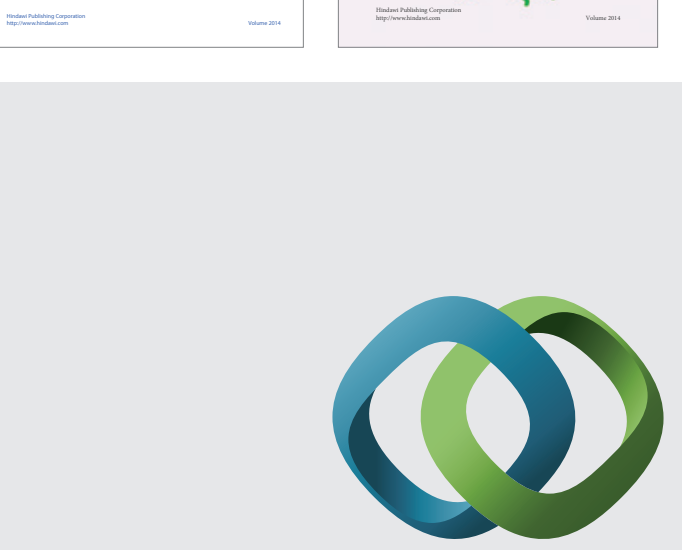

\section{Hindawi}

Submit your manuscripts at

http://www.hindawi.com
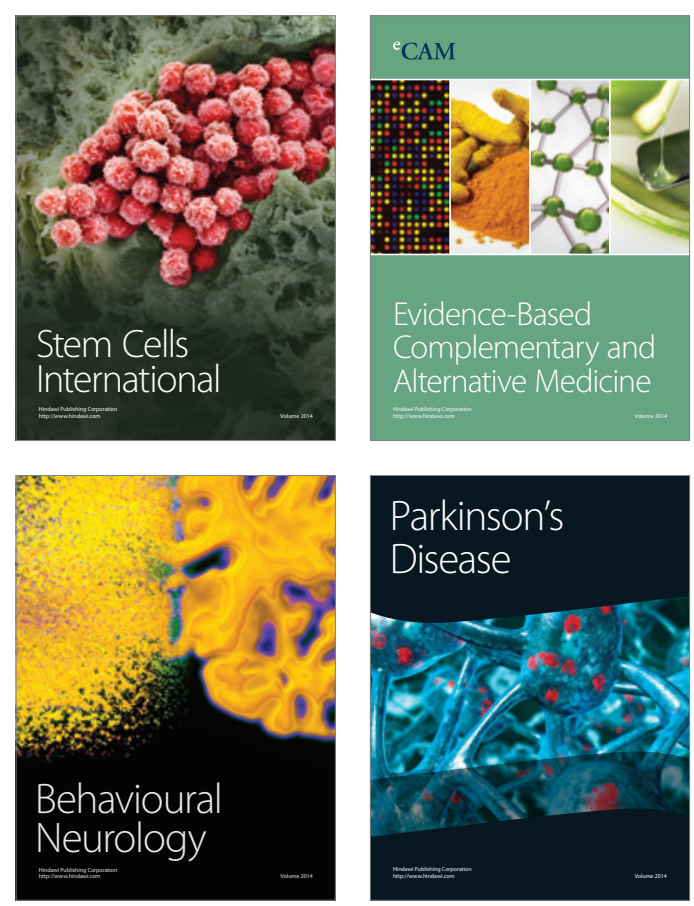

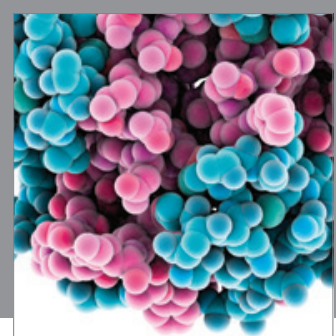

Journal of
Diabetes Research

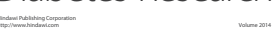

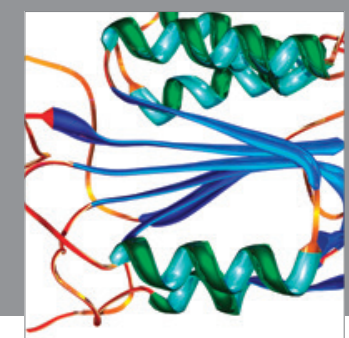

Disease Markers
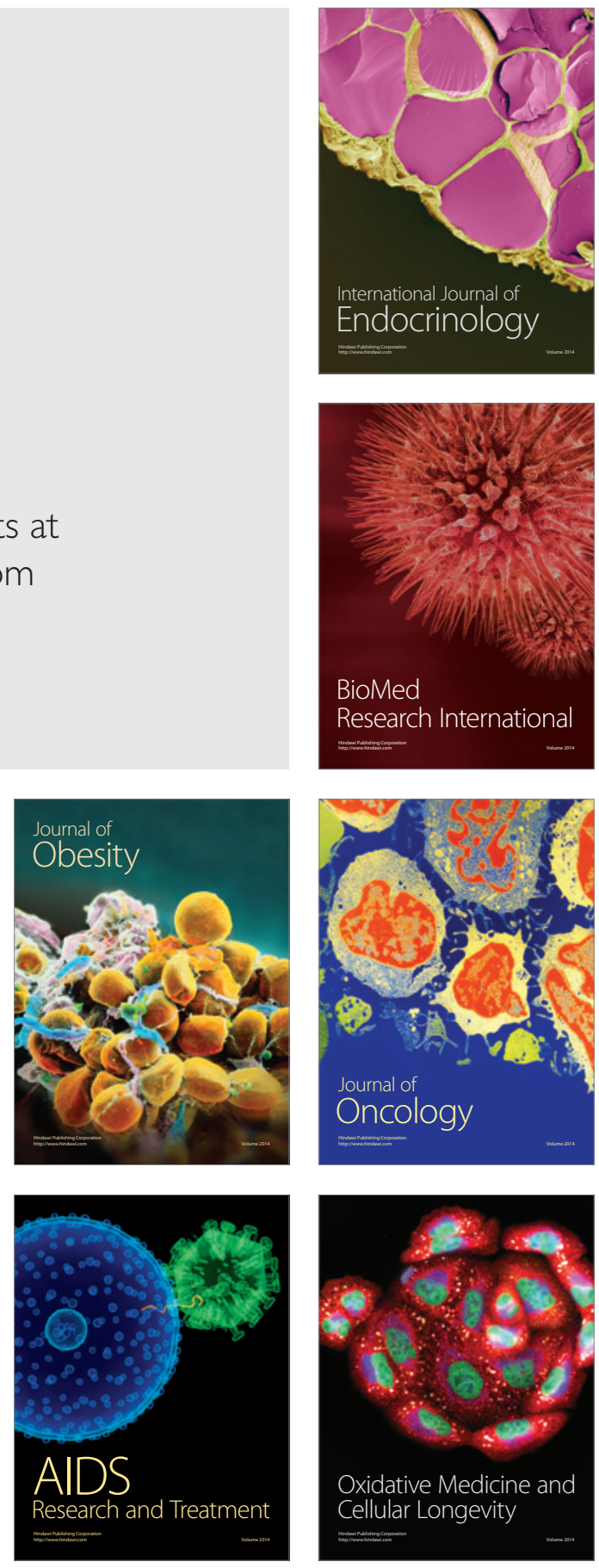